\title{
The Relationship Between the Evolution of an Internal Structure and Drug Dissolution from Controlled-Release Matrix Tablets
}

\author{
Piotr Kulinowski, ${ }^{1,4}$ Wiktor Hudy, ${ }^{1}$ Aleksander Mendyk, ${ }^{2}$ Ewelina Juszczyk, ${ }^{2}$ Władysław P. Węglarz, ${ }^{3}$ \\ Renata Jachowicz, ${ }^{2}$ and Przemysław Dorożyński ${ }^{2}$
}

Received 12 June 2015; accepted 19 August 2015; published online 4 September, 2015

\begin{abstract}
In the last decade, imaging has been introduced as a supplementary method to the dissolution tests, but a direct relationship of dissolution and imaging data has been almost completely overlooked. The purpose of this study was to assess the feasibility of relating magnetic resonance imaging (MRI) and dissolution data to elucidate dissolution profile features (i.e., kinetics, kinetics changes, and variability). Commercial, hydroxypropylmethyl cellulose-based quetiapine fumarate controlled-release matrix tablets were studied using the following two methods: (i) MRI inside the USP4 apparatus with subsequent machine learning-based image segmentation and (ii) dissolution testing with piecewise dissolution modeling. Obtained data were analyzed together using statistical data processing methods, including multiple linear regression. As a result, in this case, zeroth order release was found to be a consequence of internal structure evolution (interplay between region's areas-e.g., linear relationship between interface and core), which eventually resulted in core disappearance. Dry core disappearance had an impact on (i) changes in dissolution kinetics (from zeroth order to nonlinear) and (ii) an increase in variability of drug dissolution results. It can be concluded that it is feasible to parameterize changes in micro/meso morphology of hydrated, controlled release, swellable matrices using MRI to establish a causal relationship between the changes in morphology and drug dissolution. Presented results open new perspectives in practical application of combined MRI/dissolution to controlled-release drug products.
\end{abstract}

KEY WORDS: dissolution test; image analysis; machine learning; magnetic resonance imaging (MRI); quetiapine fumarate.

\section{INTRODUCTION}

An understanding of drug release phenomena is crucial for the interpretation of properties and behavior of dosage form in vitro and in vivo. Despite imperfections, drug dissolution methods are among the most efficient and versatile techniques for evaluation of pharmaceutical formulations. The richness and diversity of dissolution equipment, as well as experimental conditions, provide a wide range of their applications at the development stage of a medicinal product, in quality control, post-approval changes, or as a surrogate for in vivo studies (1). The power of these techniques stems from their great sensitivity to variations in dosage form properties, which are reflected in changes of dissolution profile. Unfortunately, a similar, measurable effect may be caused by various

\footnotetext{
${ }^{1}$ Institute of Technology, The Pedagogical University of Cracow, ul. Podchorążych 2, 30-084, Kraków, Poland.

${ }^{2}$ Department of Pharmaceutical Technology and Biopharmaceutics, Pharmaceutical Faculty, Jagiellonian University, Medical College, ul. Medyczna 9, 30-688, Kraków, Poland.

${ }^{3}$ Department of Magnetic Resonance Imaging, Institute of Nuclear Physics PAN, ul. Radzikowskiego 152, 31-342, Kraków, Poland.

${ }^{4}$ To whom correspondence should be addressed. (e-mail: pkulino@up.krakow.pl)
}

physicochemical factors, which significantly decreases specificity of dissolution tests $(2,3)$. Therefore, there is a need to combine dissolution methods with other techniques to improve their specificity and to further elucidate the mechanisms of drug dissolution. In the recent decades, the application of combined methods that integrate drug dissolution and imaging techniques has increased noticeably (3-12).

One of the prominent imaging techniques is magnetic resonance imaging (MRI). Microstructural properties of controlled-release dosage forms as observed by MRI correspond to molecular properties/behavior of water and its interaction with matrix components. During matrix hydration, water concentration within the matrix as well as its interaction with polymer molecules can result in the formation of specific zones or layers $(9,13-16)$.

Despite the growing number of articles presenting drug dissolution and imaging data, there is a lack of studies that directly relate the evolution of internal structures occurring in hydrating polymeric matrices with the drug release process. An extensive literature review has shown that the complicated relationship between dissolution and imaging data is not fully understood. Some elements of such relationships that suggest the complicated interplay of structural and physicochemical factors during drug dissolution are discussed by Tajarobi et al. (17). 
An equally important issue is the variability of drug dissolution in relation to the imaging data. Only a few papers in the field, present results of the systematic image analysis; however, the relationship between variability of drug dissolution results and measurements of internal structure parameters has not been fully discussed $(18,19)$. It is also surprising that there is a lack of publications presenting the results of MR imaging of multiple independent samples.

The presented study aimed to:

- Present and discuss the results of the MRI measurements of multiple independent samples of hydrated controlledrelease dosage form in relation to drug dissolution results.

- Introduce machine learning-based image analysis and quantification of images of hydrated drug delivery systems, as well as a short discussion on image analysis strategies.

\section{MATERIALS AND METHODS}

As a model dosage form, commercial controlled-release tablets (Seroquel XR $400 \mathrm{mg}$, AstraZeneca) containing quetiapine fumarate (QTP) and excipients, i.e., hypromellose (HPMC), lactose monohydrate, microcrystalline cellulose, sodium citrate, and magnesium stearate, were used.

The dissolution study was carried out using the USP apparatus 4 (DFZ 60, Erweka GmbH, Germany) equipped with an USP 4 compliant piston pump (HKP 60, Erweka $\mathrm{GmbH}$, Germany) and 22.6-mm ID cells. The experiments were performed in $1000 \mathrm{~mL}$ of distilled water circulating in a closed loop. The flow rate was $10 \mathrm{~mL} / \mathrm{min}$; the temperature in the dissolution cell was maintained at $37 \pm 0.5^{\circ} \mathrm{C}$. Five milliliters; aliquots were taken every $0.5 \mathrm{~h}$ and the extracted volume was replaced by a blank medium. The concentration of dissolved QTP was determined by measuring UV absorbance at $289 \mathrm{~nm}$ (V-530 spectrophotometer Jasco, Japan). Dissolution experiments were carried out in triplicate. Mean values of dissolved QTP as well as their standard deviations (SD) and relative standard deviations (RSD) were calculated.

KinetDS software (20) was used for modeling the dissolution curve and establishing the possible time points, at which dissolution kinetics switch. Piecewise mode was employed with an approximation accuracy, i.e., a tolerance criterion, of 0.99 and nonlinear regression enabled. All available models in the KinetDS library were employed in this analysis.

MRI was performed in a nonmagnetic flow-through cell at $4.7 \mathrm{~T}$ research system (MARAN DRX, Oxford Instruments, UK) using a flow-compensated spin-echo imaging pulse sequence. Details of the experimental setup and protocol have been described previously (14). The images were acquired during $6 \mathrm{~h}$ with a temporal resolution of $15 \mathrm{~min}$. MRI experiments were carried out for three different samples.

The image segmentation was performed using a Trainable Weka Segmentation (TWS) plug-in module (http://fiji.sc/ Trainable_Weka_Segmentation), part of the public domain software, Fiji distribution (21) of ImageJ v. 1.44 (National Institutes of Health, http://rsb.info.nih.gov/ij/). The TWS relies on algorithms included in Waikato Environment for Knowledge Analysis (WEKA) data mining and machine learning toolkit. Briefly, three separate regions in the matrix were assumed and they were segmented using methods based on image intensity and texture. Central sagittal images (Fig. 1a) of each hydrated tablet obtained at consecutive time points were stacked. Based on previously acquired knowledge, three regions inside the hydrated matrix plus region outside the matrix, which contained mainly dissolution medium, were assumed $(14,22)$. Representative samples of these regions originating from different images in the stack were manually selected for classifier training purposes (an example is presented in Fig. 1b).

All available training features (filters) were applied to the original image. The Fast Random Forest algorithm supported by WEKA was used as a classifier. The classifier was trained iteratively and finally was applied to the stacks to obtain continuous segmented regions-an example is presented in Fig. 1c, d. Figure 1e-h shows probability maps of the segmented regions. On the basis of segmentation results, areas of dry core, interface, and gel-like regions were obtained. Mean values of areas at each hydration time points as well as their standard deviations (SD) and relative standard deviations (RSD) were calculated.

Finally, multiple linear regression was carried out using $\mathrm{R}$ statistical environment routine $\operatorname{lm}($ ) (23). The independent variables were MRI and subsequent image analysis results, namely gel-like, interface, and core areas. The dependent variable was the amount of drug dissolved at each specific time point. A time variable was not introduced; instead, each case represented a particular/individual time point. Missing values of independent variables were filled by means of interpolation of the curves over the time domain using $\mathrm{R}$ statistical environment pchip() routine of the "signal" package. A direct analysis of $\operatorname{lm}()$ and its summary() routine was performed to establish the input variables' importance based on the $t$ statistic and the corresponding $p$ values computed for all coefficients.

\section{RESULTS}

Figure 2 shows dissolution and MRI data analysis results.

The dissolution of the drug over the course of the experiment was incomplete (Fig. 2a). At the sixth hour, about $21 \%$ of QTP was released. The analysis of the dissolution data with KinetDS software revealed a change in the kinetics of drug release at the fourth hour. This time point was selected automatically by the KinetDS software independent of the user. Piecewise mode of KinetDS works internally with three points assumed as a minimum of each of the "pieces" into which the original curve is dissected. Thus, the resulting switch-point should be interpreted as a range-covering point assessed by KinetDS and three preceding time points. In our case, this resulted in the range of 3.25-4.0 h. Dissolution in the time range of 0 to $4 \mathrm{~h}$ conformed to zeroth order kinetics, whereas beyond $4 \mathrm{~h}$, it changed to nonlinear. As it is presented in Fig. 3, the dissolution rate was constant and strongly fitted a linear relationship $\left(R^{2}=0.9915\right)$ between the dry core area and the interface area evolution (up to $3.5 \mathrm{~h}$ of hydration/dissolution).

According to our earlier experience, three regions in the structure of hydrated Seroquel XR matrix tablets in MRI images were identified $(14,22)$ - the core in the center of the matrix, the external gel-like layer, and the interface area between them. The interface region could be also identified as a swollen glassy zone. Figure $2 \mathrm{~b}$ shows the average results 


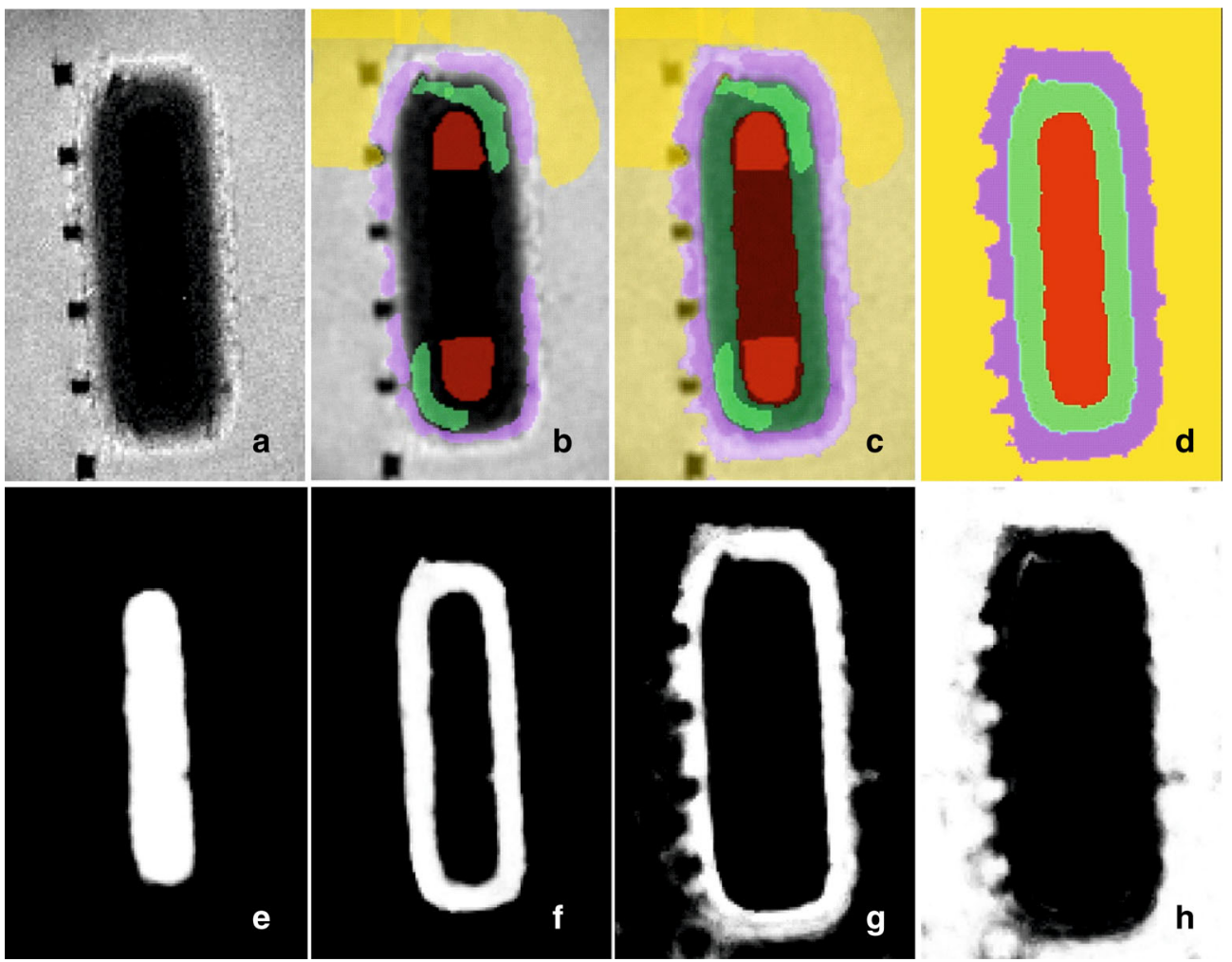

Fig. 1. Diagram of image analysis procedure. a Original image obtained at $2 \mathrm{~h}$ of hydration, b manual selection of a region's samples for training purposes (red-core, green-interface region, magenta-gel-like layer, yellow-medium and holder). $\mathbf{c}$ Result of image segmentation together with manual selection, $\mathbf{d}$ final result of segmentation. $\mathbf{e}-\mathbf{h}$ Probability maps for dry core, interface region, gel-like layer, and region outside the tablet (water and holder) respectively

of the evolution of each region by means of its area, obtained from three MRI measurements of independent samples. The change of the overall cross-section area of the tablets during $6 \mathrm{~h}$ of drug dissolution was limited, it ranged from $124.2 \pm 3.7$ to $153.4 \pm 8.6 \mathrm{~mm}^{2}$. The area of the gel-like layer increased gradually from $34.3 \pm 4.7$ to $93.3 \pm 12.8 \mathrm{~mm}^{2}$. During the $4 \mathrm{~h}$ of the experiment, the interface area increased gradually at the expense of the core, reaching a maximum $\left(85.7 \pm 3.0 \mathrm{~mm}^{2}\right)$ at $3.75 \mathrm{~h}$. At this moment, the core disappeared completely and afterwards the area of the interface started to decrease.

As a result, dissolution and imaging-based data sets were obtained. Both studies were performed in the same hydrodynamic conditions. Therefore, it was possible to relate the results of both studies. In particular, the changes in the structure of the hydrated tablets corresponded to the drug dissolution profile. Multiple linear regression was carried out to evaluate functional relationships between the MRI and the dissolution results. After the input variable reduction, two independent variables, i.e., the dry core and interface areas were found to be related to the amount of dissolved drug. The resulting relationship is presented in Eq. (1), and detailed diagnostics of the linear model is presented in the Table I.

$Q=-0.38 x-0.52 y+44.32$

where $Q$ is released amount of QTP [\%], $x$ is area of interface region $\left[\mathrm{mm}^{2}\right]$, and $y$ is the area of the core $\left[\mathrm{mm}^{2}\right]$.

The goodness of fit measures presented in Table I demonstrated that Eq. (1) was a representative model of relationship between amounts of QTP released and corresponding areas of tablets' regions/layers, i.e., interface and core, derived from MR images.

The change in dissolution kinetics at approximately $3.75 \mathrm{~h}$ coincided with an abrupt change in the relationship between the gel-like area and the interface area (see Fig. 4). After a gradual increase of both parameters up to $3.75 \mathrm{~h}$, the gel-like layer area began to increase while the interface area started to decrease. This phenomenon was associated with core loss (see Fig. 2). During the initial phase of tablet hydration, the gel layer and interface expanded at the expense of the core. After core loss the area of the gel expanded at the expense of the interface zone.

At the beginning of the dissolution $(t<4 \mathrm{~h})$, the variability of the dissolution results was very low-standard deviation (SD) ranged between 0.1 and $0.3 \%$ (see Fig. 2). Nevertheless, it was noticeable after the fourth hour dissolution became more variable (SD ranged from 1.3 to $3 \%$ ). MRI results showed (Fig. 2b) that at $3.75 \mathrm{~h}$, the dry core disappeared. After this time, SD of the gel-like and the interface evolution profiles increased. For evolution of the gel-like layer, the average SD was $1.6 \mathrm{~mm}^{2}$ up to $3.75 \mathrm{~h}$. It increased from $1.1 \mathrm{~mm}^{2}$ at $3.7 \mathrm{~h}$ to $2.9 \mathrm{~mm}^{2}$ at $4 \mathrm{~h}$ and increased to $10.5 \mathrm{~mm}^{2}$ at $6 \mathrm{~h}$. The average SD between 4 and $6 \mathrm{~h}$ was $6.9 \mathrm{~mm}^{2}$. Correlation of absolute values of the variation coefficients (\%RSD) showed a strong linear relationship $\left(R^{2}>0.9\right)$ between relative standard deviations of QTP released and RSD values for particular/individual interface areas (Fig. 5). 


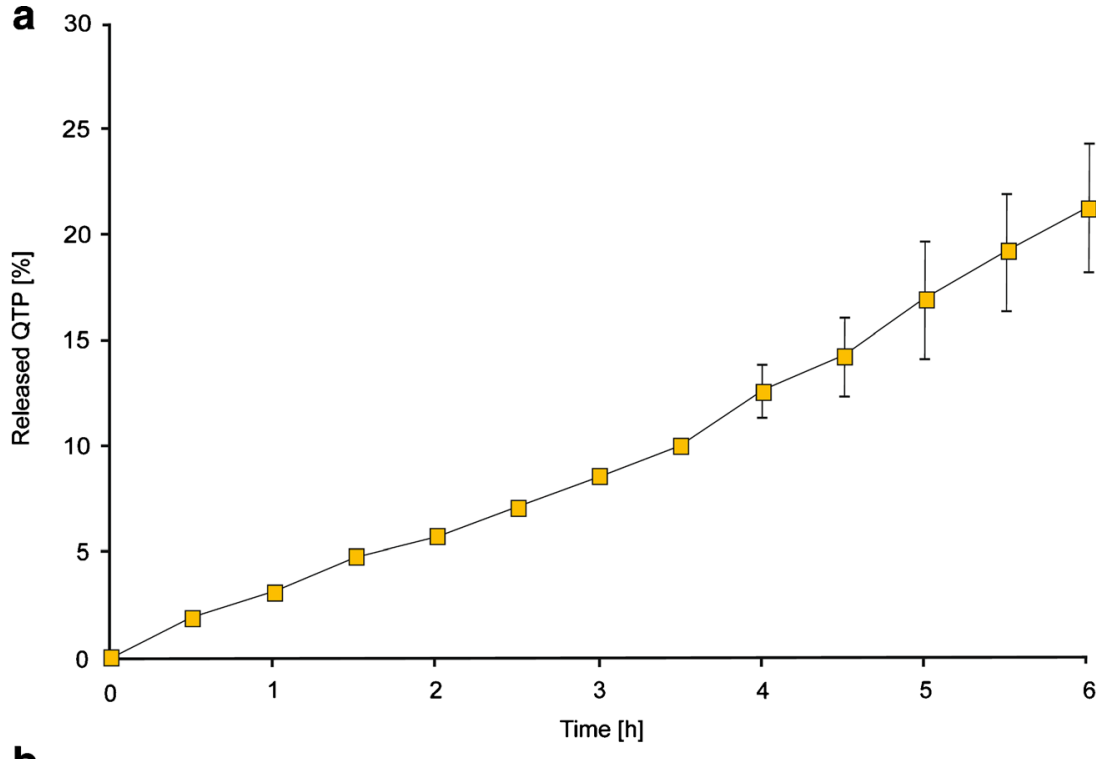

b

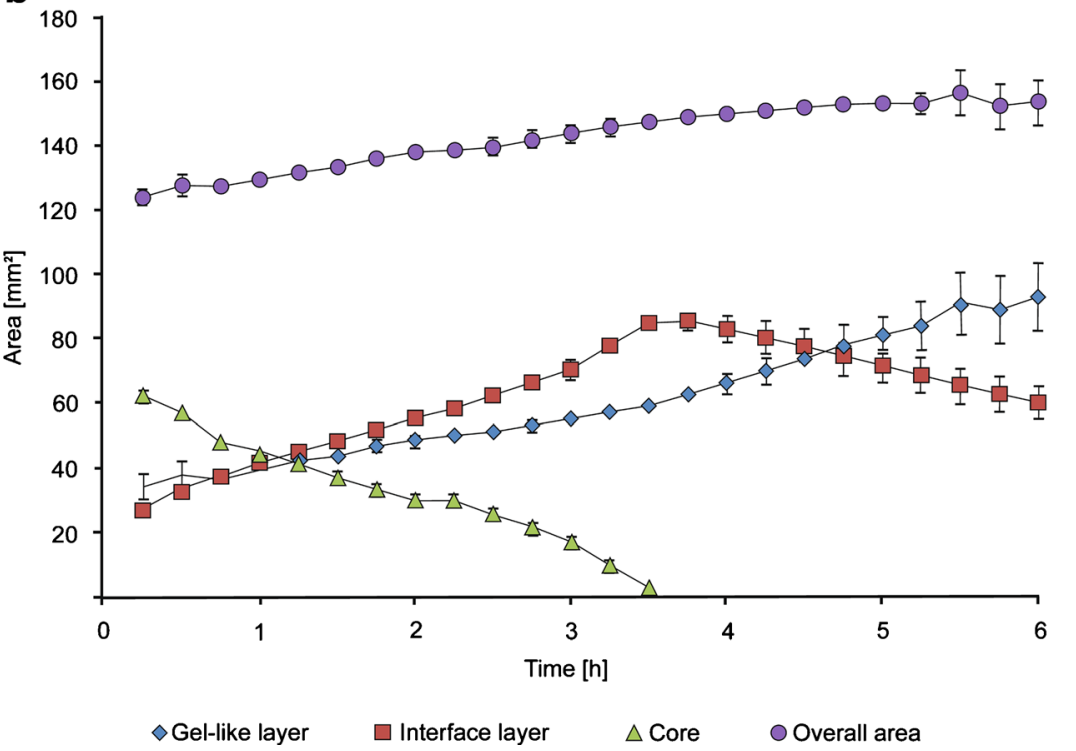

Fig. 2. a Dissolution profile of QTP $(n=3)$. Error bars represent standard deviation. b Temporal evolution of areas of individual regions inside the tablet during hydration, derived from MR images $(n=3)$. Error bars represent standard deviation

\section{DISCUSSION}

The first aspect of this study was image analysis strategies for assessment of changes in the internal structure of hydrated controlled-release matrix systems. So far, image analysis issues have only been marginally discussed in the works concerning the application of MRI. Nott (8) has proposed identification of the boundary between gel and non-hydrated core by using a contour intensity that was halfway between the highest and the lowest intensities inside the hydrated matrix - it has been established for the first image and has been consequently used throughout the series. This analysis assumed two internal matrix regions, namely the hydrated and unhydrated regions. In contrast, image intensity analysis has been applied by Kulinowski et al. to identify the existence of three regions in the hydrated matrix (14). These regions arise from three different modes in the image intensity histogram and exhibit internal inhomogeneity. In the subsequent study by Kulinowski et al., it has been demonstrated using micro-CT that this intra-region inhomogeneity is caused by the presence of air inside the hydrated part of the matrix (22). Therefore, image intensity-based segmentation (i.e., thresholding) may provide appearance of discontinuous regions as a result-the segmented regions may also contain pixels from other regions. For example, the core region segmented by thresholding contained pixels from the interface region but lacked pixels from the center of the core (14). Due to intra-region inhomogeneity and consequently to image intensity variations, it was necessary to choose an image segmentation method that take into consideration the texture of the region. To this end, in the current study, a machine learning approach using TWS was applied. It required only a selection of a representative 


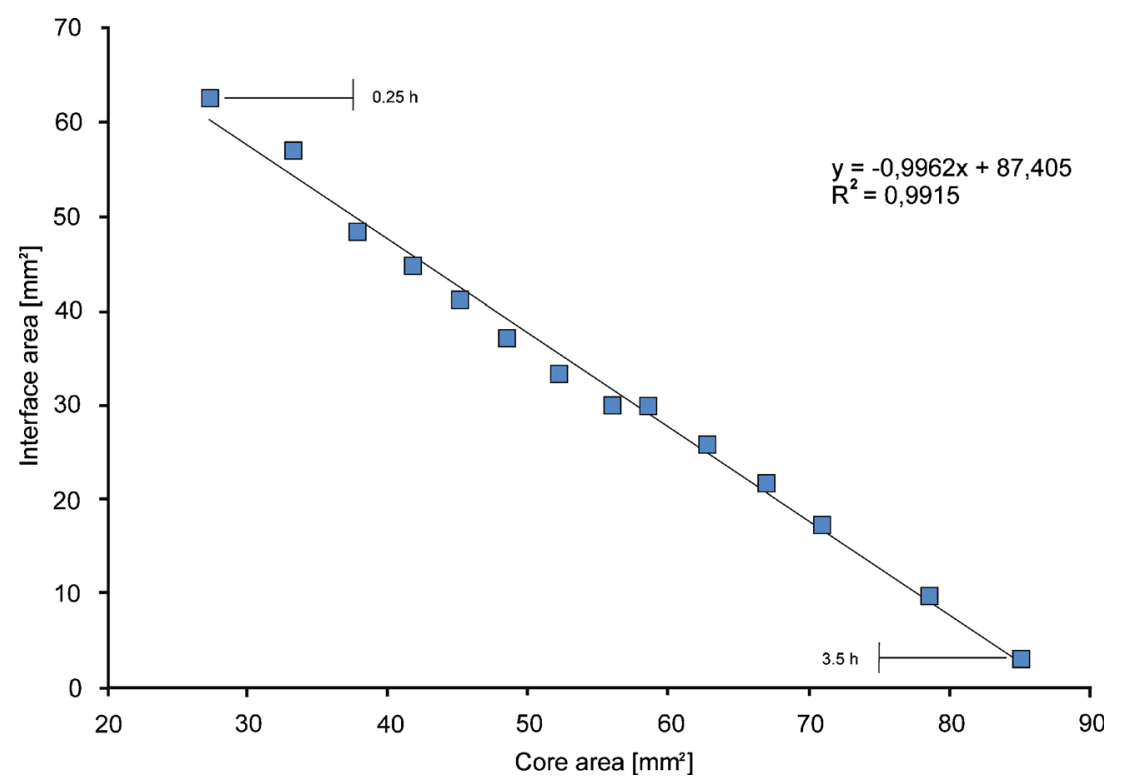

Fig. 3. Relationship between interface area and core area

region's samples (Fig. 1b). The method did not constrain the number of regions, only initial assumptions regarding the matrix structure under hydration were necessary.

The evolution of the internal structure in terms of layers in Seroquel XR by MRI has been presented for the first time by Kulinowski et al. in 2011 (14) and has been confirmed later by Kulinowski et al. in 2015 using MRI and micro-CT (22). Zhang et al. (18) have shown the evolution of the matrix in terms of gel and dry core layer thickness for the commercial modified-release pharmaceutical product Lescol XL. In the subsequent study by Chen et al. (24), dissolution profiles for Lescol XL have been related to the results of ${ }^{19} \mathrm{~F}$ imaging of active substance. However, the dissolution data has not been directly correlated with the results of ${ }^{1} \mathrm{H}$ imaging, which reflect the properties of water interacted with the matrix.

The dissolution tests allow simultaneous studies of several samples (usually six) at the same time, while the MRI can be performed for only one sample at a time. Thus, it has been

Table I. Description of Linear Model Relating Amount of QTP Released with Areas of Interface and the Core Regions as Presented in Eq. (1)

Coefficient diagnostics

\begin{tabular}{lccc}
\hline Variable & Coefficient & $\begin{array}{l}\text { Standard } \\
\text { error }\end{array}$ & $p$ value \\
\hline Intercept & 44.32 & 0.4896 & $<2 \cdot 10^{-16}$ \\
Area of interface region $(x)$ & -0.38 & 0.0063 & $<2 \cdot 10^{-16}$ \\
Area of the core region $(y)$ & -0.51 & 0.0049 & $<2 \cdot 10^{-16}$ \\
Overall equation diagnostics & & & \\
$\quad$ Residual standard error & 0.2132 & & \\
$\quad$ Root mean squared error & 0.1994 & & \\
(RMSE) & 0.9989 & & \\
$\quad R^{2}$ & 0.9988 & & \\
$\quad$ Adjusted $R^{2}$ & $<2.2 \cdot 10^{-16}$ & & \\
$p$ value $(d f=21)$ & & & \\
\hline
\end{tabular}

$Q T P$ quetiapine fumarate a common practice to present imaging results for one hydration cycle of a single sample $(3,7,14,17-19,25,26)$. For example, in the work by Zhang et al. (18), only one sample was imaged, and the layer evolution data was plotted as the mean of four layer thickness measurements, each taken in a different direction. A similar approach is presented by Punčochová et al. with regard to the MR images of tablets containing Aprepitant solid dispersions (19). In this work, the calculations of mean and SD of the front positions are based on ten measurements performed on a single image. The only exception was MRI results obtained by Nott (8) which concerned six independent samples. Alas, the author has not combined these results with those of dissolution test; therefore, their usefulness in our discussion is limited.

The relation between image features and dissolution data has been given very little attention in previous studies. Dissolution and imaging data together, obtained using a non-pharmacopoeial flow-through cell, have been presented by Tajarobi et al. (17) where HPMC matrices with mannitol as the soluble model of active substance have been tested. The results demonstrate that almost the entire dose of mannitol is released by the time the core of the HPMC/mannitol tablet had disappeared.

Therefore, presentation, analysis, and correlation of image-based evolution of the matrix with dissolution test results were the next logical step. That was the rationale for the present work: to find features relating both data sets. As described in the Results section, piecewise analysis of the dissolution profile by KinetDS resulted in a division of the profile into two parts of different characteristics. The first part showed zeroth order release, which was associated with the existence of a dry core and was achieved by proper balance between the swollen region and the dry core (see Fig. 3). Therefore, the existence of a dry core was crucial for a constant release rate. Disappearance of the dry core was accompanied by the reversal of the relationship between the gel-like and interface layers (see Fig. 4), which in turn resulted in the loss of zeroth order release kinetics. It was probably one of the most valuable observations of the study because it explained 


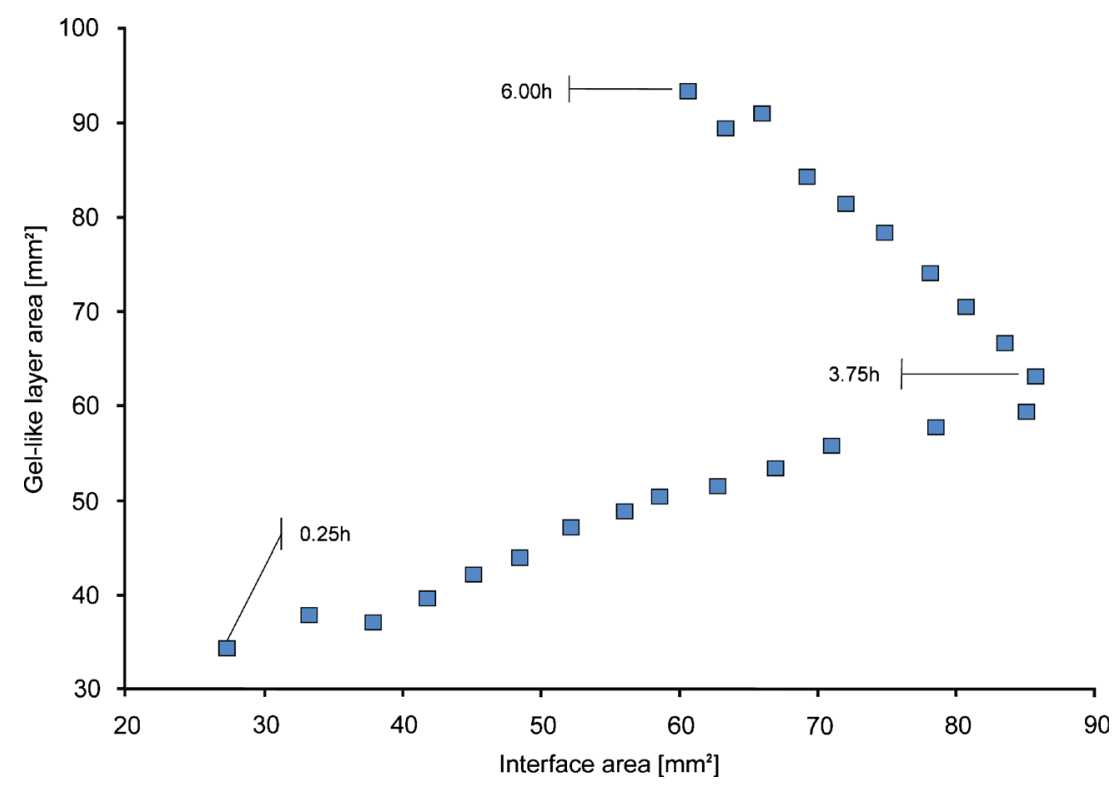

Fig. 4. Relationship between gel-like layer area and interface area reverses after $3.75 \mathrm{~h}$

the subtle effects observed in dissolution profiles using supplementary imaging. Without MRI, such changes in dissolution characteristics could be omitted or misinterpreted as results of analytical errors. Furthermore, the image analysis methods used were highly independent of the user, thus largely reducing the risk of misinterpreting the data as a consequence of user error or bias.

One of the first imaging-based approaches used to assess dissolution variability has been performed earlier by Dorożyński et al. (26). It has been carried out on commercial diclofenac modified-release formulations. It has been found that the complexity of the external matrix boundary described by fractal dimension could be one of the sources of dissolution variability.

The results of the current study demonstrated that another possible source of variability in dissolution results could be the internal structure of the hydrated tablet itself. Data presented in Fig. 2 showed that at the fourth hour, the SD of dissolution results increased with the disappearance of the dry core. On the other hand, the absence of a rigid internal structure caused an increase in variability of the cross-section areas of the gel-like and interface layers.

The variability of drug dissolution results may stem from various sources, e.g., uniformity of dosage forms, dissolution conditions, and equipment $(27,28)$. Variability in dissolution profiles is crucial for CR dosage forms due to safety and efficacy reasons. In the current work, for the first time, the sources of variability in dissolution results were addressed and were associated with changes of the internal structure of the dosage form during in vitro hydration and dissolution processes.

In standard approaches to drug dissolution, the reasons for a particular dissolution curve features remained unknown.

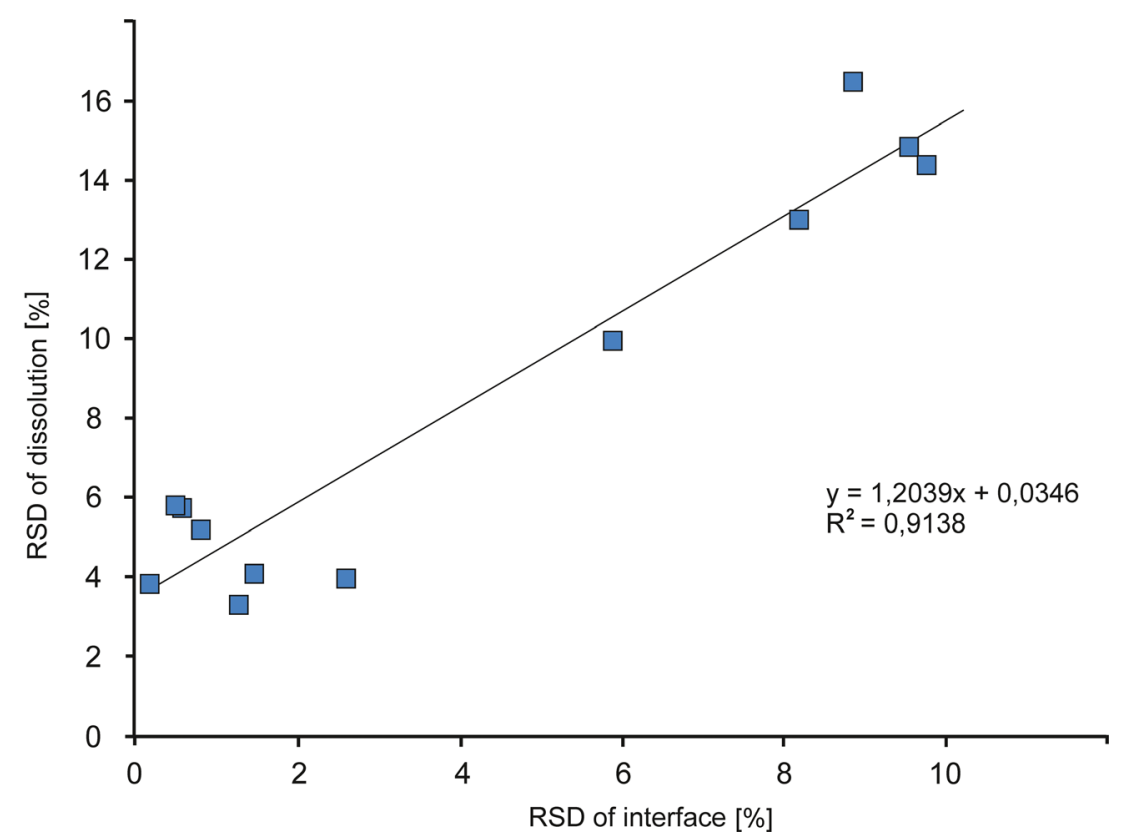

Fig. 5. Correlation between RSD values for interface region area and dissolution results 
In fact, they could be induced by various factors. In 2003, Sawada et al. elucidated the impact of some of these factors that could not otherwise be identified by in vitro dissolution test (2). The results of the current study demonstrated that properly designed imaging studies and image analysis as a supplement to dissolution data can be useful. However, it should be kept in mind that every case requires its own dedicated methodology. To be able to correlate imaging results and dissolution test results, image analysis should reflect the actual structure of the matrix. This structure as imaged in USP 4 apparatus should be confirmed using other imaging modalities, e.g., other MRI sequences or micro-CT. Such knowledge should be gained prior to the attempt to correlate dissolution and imaging-based data.

\section{CONCLUSIONS}

The results of the study showed that parameterization of the changes in micro/meso morphology of hydrated, controlledrelease swellable matrices as obtained by magnetic resonance imaging could be directly related to dissolution results. Magnetic resonance imaging inside the USP apparatus 4 in real dissolution testing conditions with subsequent machine learning-based image segmentation, drug release studies with subsequent piecewise dissolution profile modeling, and statistical data processing including multiple linear regression were used to accomplish this task. A causal relationship between matrix morphology changes and drug dissolution was revealed. Moreover, an MRI performed during dissolution testing allowed identification of potential sources of dissolution variability.

In this particular case, dry core disappearance had an impact on:

- Change in dissolution kinetics (from zeroth order to unknown).

- Variability of in vitro drug dissolution-the increase in dissolution variability in vitro coincided with dry core disappearance.

Presented results open new perspectives in practical application of combined MRI/dissolution to controlled-release drug products.

\section{ACKNOWLEDGMENTS}

The work was supported by the Polish Ministry of Science and Higher Education grant NN518 407438. The project was co-financed by the European Regional Development Fund under the Infrastructure and Environment Program UDA-POIS.13.01-023/09-00.

The authors are thankful to Prof. Greg Stanisz, Sunnybrook Health Sciences Centre, Toronto, Canada, for critical reading of the manuscript.

Open Access This article is distributed under the terms of the Creative Commons Attribution 4.0 International License (http://creativecommons.org/licenses/by/4.0/), which permits unrestricted use, distribution, and reproduction in any medium, provided you give appropriate credit to the original author(s) and the source, provide a link to the Creative Commons license, and indicate if changes were made.

\section{REFERENCES}

1. Patadia R, Vora C, Mittal K, Mashru R. Dissolution criticality in developing solid oral formulations: from inception to perception. Crit Rev Ther Drug Carrier Syst. 2013;30(6):495534.

2. Sawada T, Sako K, Fukui M, Yokohama S, Hayashi M. A new index, the core erosion ratio, of compression-coated timed-release tablets predicts the bioavailability of acetaminophen. Int J Pharm. 2003;265(1-2):55-63.

3. Dorożyński PP, Kulinowski P, Mendyk A, Młynarczyk A, Jachowicz R. Novel application of MRI technique combined with flow-through cell dissolution apparatus as supportive discriminatory test for evaluation of controlled release formulations. AAPS PharmSciTech. 2010;11(2):588-97.

4. Fyfe CA, Grondey H, Blazek-Welsh AI, Chopra SK, Fahie BJ. NMR imaging investigations of drug delivery devices using a flow-through USP dissolution apparatus. J Control Release. 2000;68(1):73-83.

5. Kulinowski P, Dorożyński P, Jachowicz R, Jasiński A. Magnetic resonance imaging analysis of moving fronts in floating dosage forms. Acta Phys Pol A. 2005;108(1):155-60.

6. Dorożyński P, Kulinowski P, Jachowicz R, Jasiński A. Development of a system for simultaneous dissolution studies and magnetic resonance imaging of water transport in hydrodynamically balanced systems: a technical note. AAPS PharmSciTech. 2007;8(1).

7. Kulinowski P, Dorożyński P, Jachowicz R, Węglarz WP. An integrated system for dissolution studies and magnetic resonance imaging of controlled release, polymer-based dosage forms-a tool for quantitative assessment of hydrogel formation processes. J Pharm Biomed Anal. 2008;48(3):685-93.

8. Nott KP. Magnetic resonance imaging of tablet dissolution. Eur J Pharm Biopharm. 2010;74(1):78-83.

9. Dorożyński PP, Kulinowski P, Młynarczyk A, Stanisz GJ. Foundation review: MRI as a tool for evaluation of oral controlled release dosage forms. Drug Discov Today. 2012;17(3-4):110-23.

10. Kazarian SG, Ewing AV. Applications of Fourier transform infrared spectroscopic imaging to tablet dissolution and drug release. Expert Opin Drug Deliv. 2013;10(9):1207-21.

11. Ostergaard J, Lenke J, Jensen SS, Sun Y, Ye F. UV imaging for in vitro dissolution and release studies: initial experiences. Dissolution Technol. 2014;21(4):27-38.

12. Smith GPS, McGoverin CM, Fraser SJ, Gordon KC. Raman imaging of drug delivery systems. Adv Drug Deliv Rev. 2015;89:21-41.

13. Kojima M, Ando S, Kataoka K, Hirota T, Aoyagi K, Nakagami H. Magnetic resonance imaging (MRI) study of swelling and water mobility in micronized low-substituted hydroxypropylcellulose matrix tablets. Chem Pharm Bull. 1998;46(2):324-8.

14. Kulinowski P, Dorożyński P, Młynarczyk A, Węglarz WP. Magnetic resonance imaging and image analysis for assessment of HPMC matrix tablets structural evolution in USP apparatus 4. Pharm Res. 2011;28(5):1065-73.

15. Kulinowski P, Młynarczyk A, Dorożyński P, Jasiński K, Gruwel MLH, Tomanek B, et al. Magnetic resonance microscopy for assessment of morphological changes in hydrating hydroxypropylmethyl cellulose matrix tablets in situ. Pharm Res. 2012;29(12):3420-33.

16. Kulinowski P, Młynarczyk A, Jasiński K, Talik P, Gruwel MLH, Tomanek B, et al. Magnetic resonance microscopy for assessment of morphological changes in hydrating hydroxypropylmethylcellulose matrix tablets in situ - is it possible to detect phenomena related to drug dissolution within the hydrated matrices? Pharm Res. 2014;31(9):2383-92.

17. Tajarobi F, Abrahmsen-Alami S, Carlsson AS, Larsson A. Simultaneous probing of swelling, erosion and dissolution by NMRmicroimaging-effect of solubility of additives on HPMC matrix tablets. Eur J Pharm Sci. 2009;37(2):89-97.

18. Zhang Q, Gladden L, Avalle P, Mantle M. In vitro quantitative $\mathrm{H}-$ 1 and F-19 nuclear magnetic resonance spectroscopy and imaging studies of fluvastatin (TM) in Lescol (R) XL tablets in a USP-IV dissolution cell. J Control Release. 2011;156(3):345-54.

19. Punčochová K, Ewing AV, Gajdošová M, Sarvašová N, Kazarian $\mathrm{SG}$, Beránek J, et al. Identifying the mechanisms of drug release 
from amorphous solid dispersions using MRI and ATR-FTIR spectroscopic imaging. Int J Pharm. 2015;483(1-2):256-67.

20. Mendyk A, Jachowicz R, Fijorek K, Dorozynski P, Kulinowski P, Polak S. KinetDS: an open source software for dissolution test data analysis. Dissolution Technol. 2012;19(1):6-11.

21. Schindelin J, Arganda-Carreras I, Frise E, Kaynig V, Longair $\mathrm{M}$, Pietzsch $\mathrm{T}$, et al. Fiji: an open-source platform for biological-image analysis. Nat Methods. 2012;9(7):676-82.

22. Kulinowski P, Woyna-Orlewicz K, Rappen G-M, HaznarGarbacz D, Węglarz WP, Dorożyński PP. An understanding of modified release matrix tablets behavior during drug dissolution as the key for prediction of pharmaceutical product performance - case study of multimodal characterization of quetiapine fumarate tablets. Int J Pharm. 2015;484(1-2):235-45.

23. R Core Team, R: a language and environment for statistical computing, R Foundation for Statistical Computing, Vienna, Austria, 2014 [2015.05.12]. Available from: http://www.R-project.org.
24. Chen C, Gladden LF, Mantle MD. Direct visualization of in vitro drug mobilization from lescol XL tablets using two-dimensional F-19 and H-1 magnetic resonance imaging. Mol Pharm. 2014;11(2):630-7.

25. Viriden A, Abrahmsen-Alami S, Wittgren B, Larsson A. Release of theophylline and carbamazepine from matrix tablets - consequences of HPMC chemical heterogeneity. Eur J Pharm Biopharm. 2011;78(3):470-9.

26. Dorożyński P, Kulinowski P, Jamróz W, Juszczyk E. Geometry of modified release formulations during dissolution-influence on performance of dosage forms with diclofenac sodium. Int $\mathbf{J}$ Pharm. 2014;477(1-2):57-63.

27. Elkoshi Z. On the variability of dissolution data. Pharm Res. 1997;14(10):1355-62.

28. Qureshi SA, Shabnam J. Cause of high variability in drug dissolution testing and its impact on setting tolerances. Eur J Pharm Sci. 2001;12(3):271-6. 